\title{
PREVALENCIA DE CARIES DENTAL E ÍNDICE CPOD EN ESCOLARES DE 12 AÑOS EN LA PARROQUIA BAÑOS DEL CANTÓN CUENCA 2016
}

\section{PREVALENCE OF DENTAL CARIES AND DMFT INDEX IN 12 YEARS OLD SCHOOL CHILDREN AT BAÑOS DISTRICT OF CUENCA 2016}

\author{
Flores-Tenechagua Magalli $^{1 *}$, Villavicencio-Caparó Ebingen ${ }^{2}$, Corral-Peñafiel Davíd ${ }^{2}$ \\ ${ }^{1}$ Odontóloga egresada de la Universidad Católica de Cuenca. Ecuador \\ ${ }^{2}$ Dr. Mg. Esp Coordinador de investigación de la Carrera de Odontología, Universidad Católica de Cuenca. Ecuador \\ 3 Odontólogo egresado de la Universidad Católica de Cuenca. Ecuador \\ *magaflo61@ hotmail.com
}

\begin{abstract}
Resumen
OBJETIVO: Determinar la prevalencia de caries dental en escolares de 12 años de la Parroquia Baños del Cantón Cuenca 2016. MATERIALES Y MÉTODOS: Se realizó un estudio descriptivo transversal observacional teniendo en cuenta 104 pacientes con sus respectivas fichas epidemiológicas de escolares de 12 años cumplidos o por cumplir hasta diciembre 2016, los datos fueron tomados por estudiantes debidamente calibrados por un Epidemiólogo experto en el tema y a su vez ingresados a una base de datos en el programa EPIINFO ver 7.2, quienes reflejaron información epidemiológica del año 2016. RESULTADOS: La prevalencia de caries fue de $67,31 \%$ y sanos de 32,69\%, la prevalencia fue más significativa en mujeres con un porcentaje de $52,86 \%$ que en hombres que presentaron una prevalencia de 47,14\%; el índice CPOD poblacional en los escolares fue de 3,69 el cual no tuvo diferencias significativas entre el sexo masculino y femenino presentando valores de CPOD entre $(3,89$ - 3,55) respectivamente. La severidad en los escolares presentó un 31,73\%; brindando una severidad "Muy Baja" de enfermedad de caries y el grupo dentario con mayor prevalencia de caries fueron los primeros molares inferiores, con un porcentaje en la pieza dentaria 4,6 de un $16 \%$ y en la pieza dental 3,6 un 13\%. CONCLUSIONES: En los resultados obtenidos se concluyó que la prevalencia de caries en escolares de 12 años es elevada, ya que más de la mitad de los escolares la presentaban, con una mayor incidencia en el sexo femenino.
\end{abstract}

Palabras clave: Prevalencia, caries dental, índice CPOD.

\begin{abstract}
AIM Determine the prevalence of dental decay and DMFT in 12-year-old school children at Baños district of the city of Cuenca. MATERIALS AND METHODS: A descriptive cross-sectional study was carried out, taking into account 104 epidemiological records of 12-year-old students. Students duly calibrated by an expert epidemiologist. The data taken was enrolled in a database in the EPIINFO program version 7.2, which reflected epidemiological information of the year 2016. RESULTS: The prevalence of caries was $67.31 \%$ and non caries $32.69 \%$, the prevalence was more significant in women with a percentage of $52.86 \%$ than in men presenting a prevalence of $47.14 \%$; The DMFT index in the students was 3.69, which did not have significant differences between male and female, showing values of DMFT between (3.89 and 3.55), respectively. The severity in the students presented a $31.73 \%$; providing a "very low" severity of caries disease. The dental group with the highest prevalence of caries was the first lower molars, with percentage of tooth number 4.6 of $16 \%$ and tooth number 3.6 of $13 \%$. CONCLUSIONS: The prevalence of caries in 12 years school children is high, since more than half presented it, with a higher incidence in females.
\end{abstract}

Key words: Prevalence, dental caries, CPOD index. 


\section{INTRODUCCIÓN}

La evaluación periódica de la prevalencia de caries y del índice CPOD, es necesaria para que los estados puedan planificar sus servicios dentales, tanto en el sector público como en el privado. La caries dental es una enfermedad infecciosa, multifactorial, dinámica, que junto con las enfermedades periodontales son consideradas como las causas de mayor compromiso en la morbilidad bucal. ${ }^{1,2}$ La caries es una enfermedad de avance rápido y si no recibe un tratamiento oportuno seguirá propagándose e influirá en la salud general del niño causando dolor y por consiguiente ausentismo escolar. ${ }^{2-4} \mathrm{Al}$ ser detectada a tiempo esta enfermedad, se evita su cronicidad y posterior extracción de la pieza dental afectada; reduciendo así el número de extracciones dentarias que se deberá realizar en los centros de salud, consiguiendo la disminución del gasto público a nivel odontológico. ${ }^{1}$ En Ecuador, el área mínima de gestión política territorial se denomina parroquia. En la actualidad existen pocos reportes respecto a la situación de salud bucal en nuestra comunidad, por lo que se realizó un estudio descriptivo epidemiológico, para determinar la magnitud del problema de salud bucal en la parroquia Baños de la ciudad de Cuenca y a su vez esto sirva de apoyo al Ministerio de Salud Pública para conocer el estado actual de la prevalencia de caries.

Por lo tanto esta investigación plantea responder a la pregunta ¿Cuánto es la prevalencia de caries dental? Y para obtener respuesta nos enmarcamos dentro de la especialidad de cariología en la línea de investigación de la frecuencia de la enfermedad.

\section{MATERIALES Y MÉTODOS}

Para responder a la pregunta de investigación, se evaluaron a escolares de 12 años que presentaron el consentimiento de los padres y también su asentimiento informado; así mismo al finalizar el examen bucal a los escolares se les indicó su diagnóstico para motivarlos a recuperar su salud dental. Se calculó la muestra mediante fórmula presentada por (Villavicencio E) 2016. ${ }^{7,12}$

$$
n=\frac{\left(Z^{2} \cdot p \cdot q \cdot N\right)}{Z^{2} \cdot p \cdot q+E^{2}(N-1)} ;
$$

La cual fue de 104 pacientes, luego se estratificó proporcionalmente de acuerdo al tamaño de cada colegio. Para el registro de los datos, se tomó en cuenta las fichas epidemiológicas de la parroquia "Baños", las cuales fueron ingresadas a una base de datos en el programa EPIINFO versión;, ${ }^{2,7}$ reflejaron información epidemiológica de los escolares evaluados en el macro proyecto Mapa Epidemiológico de Salud bucal Cuenca 2016. El estudio de caries dental buscó describir cuantitativamente el problema en escolares de 12 años de edad, utilizando las recomendaciones de la Organización Mundial de la Salud (OMS) ${ }^{13}$ para diagnosticar la presencia de caries dental que se detalla a continuación:

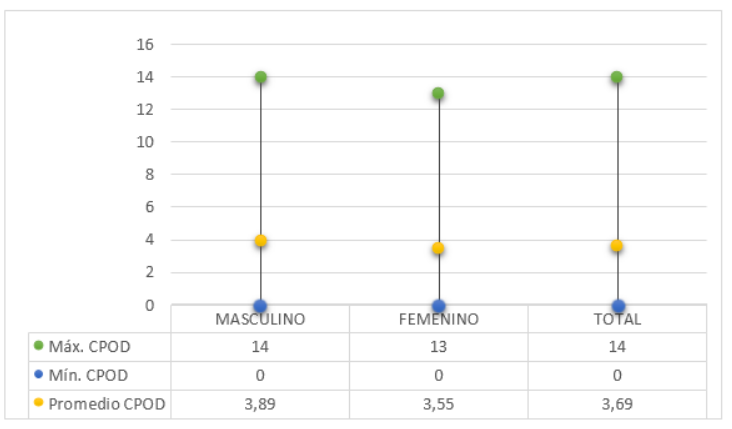

Grf. 1. Índice CPOD de acuerdo al sexo en escolares de 12 años de la Parroquia Baños, 2016.

Los examinadores fueron estudiantes de la Facultad de Odontológica de quinto año, debidamente calibrados por un epidemiólogo experto en el tema; para el examen bucal realizado a los niños de 12 años el investigador tuvo que empezar por "observar el 3er molar superior derecho (diente 1.8), continuando con el segundo molar superior derecho (1.7), luego la pieza dental 1.6 y así sucesivamente hasta llegar al 3er molar superior izquierdo (2.8), con el cual terminará los dientes superiores. Luego continuó con los dientes inferiores, examinando el 3er molar inferior izquierdo (número 3.8), luego la pieza dental 3.7, después la pieza dental 3.6 y así cada uno de los dientes inferiores hasta terminar en el 3er molar inferior derecho (número 4.8). Después de revisar cada cuadrante el examinador y el anotador tuvieron que corroborar que los datos hayan sido correctos utilizando la palabra CORRECTO". ${ }^{14}$

El examinador siguió las siguientes recomendaciones: ${ }^{14}$

1) En lo posible no tocar la boca del paciente con los dedos. Inicialmente, cada diente se examinó en forma visual para observación de áreas descalcificadas, opacidades de los bordes marginales y esmalte socavado en fosas y fisuras.

2) En pacientes que presenten extracciones dentales indagar el motivo de dicho procedimiento, pero si la respuesta no permite obtener una conclusión el examinador seguirá su criterio clínico.

3) Dictar el código claramente, para evitar, errores de anotación. Se debe interrumpir tantas veces sea necesario para hacer aclaraciones sobre una anotación.

4) Se deben examinar todas las superficies del diente.

Para la aplicación de algunos criterios, al momento del examen, es aconsejable tener en cuenta la secuencia de erupción dentaria. Se consideró como erupcionado un diente deciduo o permanente cuando cualquier porción de su corona clínica ha atravesado la fibro mucosa gingival y puede ser tocado con la sonda periodontal. Cuando se realizó el examen, los datos se consignaron 


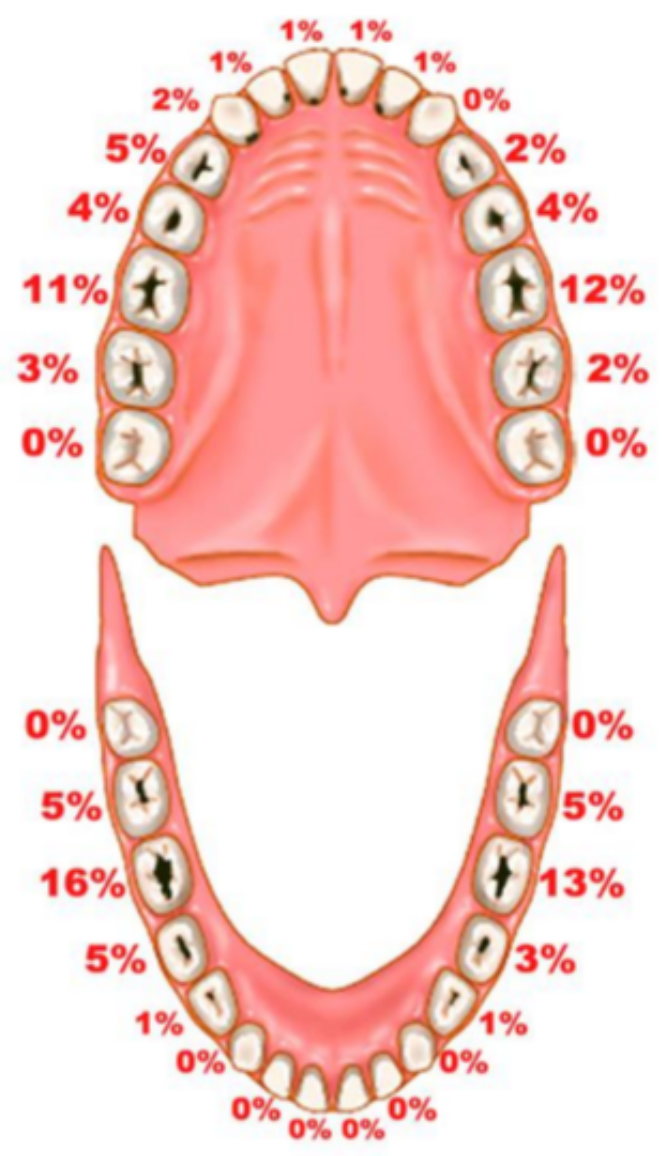

Grf. 2. Prevalencia de caries por grupo dentario en escolares de 12 años de la Parroquia Baños, 2016.

en las casillas correspondientes, anotando el código que representa el criterio de clasificación o denominación del hallazgo clínico o del tipo de tratamiento requerido, según los respectivos códigos. ${ }^{13,14}$

- Código 0- Diente Sano

- Código 1- Diete cariado

- Código 2- Diente obturado con caries

- Código 3- Diente obturado sin caries

- Código 4- Diente perdido por caries

- Código 5- Diente perdido por otro motivo

- Código 6- Presencia de sellante

- Código 7- Pilar de puente, corona o implante

- Código 8- Diente permanente sin erupcionar

- Código 9- No registrable

\section{RESULTADOS}

Se evaluaron 104 escolares de 12 años de la parroquia Baños del Cantón Cuenca, siendo 44 del sexo masculino y 60 del sexo femenino. La prevalencia de caries fue de un $67,31 \%$ y los escolares sanos fueron un $32,69 \%$. Gráfico 1 Además la prevalencia de caries respecto al sexo en los

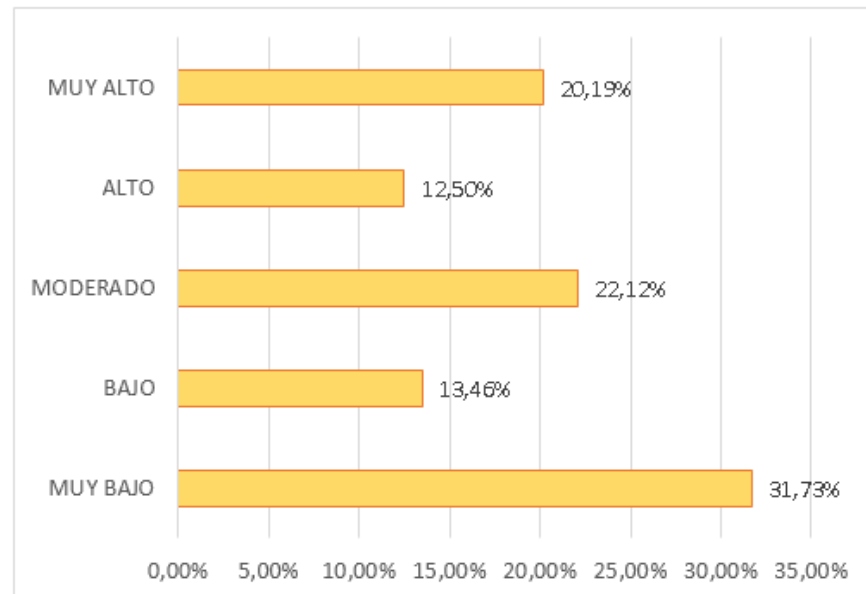

Grf. 3. Niveles de severidad del CPOD en los escolares de 12 años de la parroquia baños, 2016.

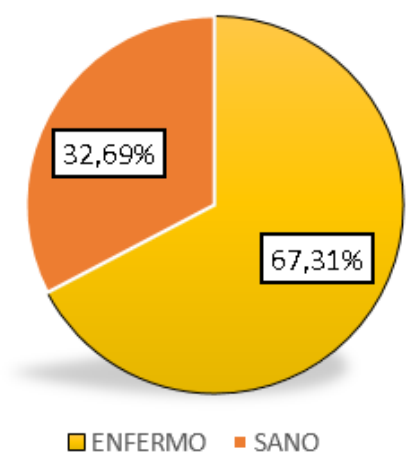

Grf. 4. Prevalencia de caries en escolares de 12 años de la Parroquia Baños, 2016.

escolares de la Parroquia Baños fue más significativa en el sexo femenino con un $52,86 \%$ que en el sexo masculino con un $47.14 \%$. Tabla 1

El índice CPOD poblacional en los escolares fue de 3,69; el cual no tuvo diferencias significativas entre el sexo masculino y femenino; presentando valores entre $(3,89-3,55)$ respectivamente. Gráfico 2

Encontramos la presencia de una severidad "Muy Baja" con un porcentaje de $31.73 \%$ brindándonos la información que en la mayoría de niños de nuestra población presentan pocas caries. Gráfico $3 \mathrm{La}$ prevalencia de caries de acuerdo al grupo dentario, se determinó que los primeros molares inferiores presentaron más caries en comparación con el resto de piezas dentarias, con un porcentaje en la pieza dentaria 4,6 de un $16 \%$ y en la pieza dental 3,6 un $13 \%$. Gráfico 4 


\begin{tabular}{|c|c|c|c|c|c|c|}
\hline \multicolumn{7}{|c|}{ SEXO } \\
\hline & \multicolumn{2}{|c|}{ MASCULINO } & \multicolumn{2}{|c|}{ FEMENINO } & \multirow[b]{2}{*}{ Total } & \multirow[b]{2}{*}{$\%$} \\
\hline & $\mathbf{n}$ & $\%$ & $\mathbf{N}$ & $\%$ & & \\
\hline SANO & 11 & $32.35 \%$ & 23 & $67.65 \%$ & 34 & $100 \%$ \\
\hline ENFERMO & 33 & $47.14 \%$ & 37 & $52.86 \%$ & 70 & $100 \%$ \\
\hline
\end{tabular}

Tabla 1. Prevalencia de caries dental en escolares de 12 años de acuerdo al sexo de la Parroquia Baños, 2016.

\section{DISCUSIÓN}

El presente estudio se basó de manera primordial en la determinación de la prevalencia de caries en escolares de 12 años, se escogió esta edad, debido a la existencia del recambio dentario, existiendo un resultado del $67,31 \%$ de caries y el índice CPOD de 3,69, éste resultado tuvo similitud con los estudios realizados en Chile por Fernández González, ${ }^{5}$ en el año 2011 y Pumalema- Saltos ${ }^{6}$ en Ecuador en el año 2011 quienes obtuvieron una prevalencia de caries de un $63.9 \%$ y $58,21 \%$ respectivamente y un índice CPOD de 3,15 en Chile y 3,48 en Ecuador.

Conflicto de intereses y financiamiento Los autores declaran no tener conflicto de intereses, haber cumplido con los requisitos de autoría y haber autofinanciado este artículo.

\section{Referencias}

1 Longás B. Estudio sobre la caries en una población infantil en la Comunidad de Madrid año 2009. [Tesis Doctoral]. Universidad de Sevilla. España; 2011 Documento disponible

2 Cerón X. El sistema ICDAS como método complementario para el diagnóstico de caries dental. Rev. CES Odont. 2015; 28 (2): 1-12.Documento disponible

3 Padilla J. Diagnóstico de salud bucodental en niños de 6 a 12 años de edad del nivel primaria en la ciudad de Tampico, Madero y Altamira. [Tesis Doctoral]. Universidad de Sevilla. España; 2014.Documento disponible

4 Fernández M. Estudio de la Caries Dental como Factor de Riesgo de Urgencias Estomatológicas en niños de 2 a 5 años En el policlínico 27 de Noviembre del municipio Marianao 2006-2007. [Tesis Doctoral] Instituto Superior de la Habana. Facultad de Estomatología; 2007 Documento disponible

5 Fernández C, Núñez L y cols. Determinantes de salud oral en población de 12 años. Rev. Clínica de Periodoncia, Implantología y Rehabilitación Oral.2011. 4(3); 117-121.
6 Pumalema F. Prevalencia de caries en los escolares de 6 a 12 años de edad de la escuela fiscal mixta Abdón Michelena de la ciudad de quito en el período lectivo 2010 - 2011. Tesis Pregrado. Universidad Central del Ecuador. 2011. Documento disponible

7 Ulate J, Rodríguez A y cols. Prevalencia De Caries Dental En Niños Y Niñas Escolares De 12 Años De Edad En Costa Rica, 2006. Rev. Científica Odont. 2011. 7(2).

8 Reinoso N, Villavicencio E. Caries dental en escolares de 12 años de sayausí,cuenca, ecuador. Revista OACTIVA, Vol 1 No1 2016. Documento disponible

9 Coronado C, Riquelme J. Estado de salud de los primeros molares permanentes en niños de 12 y 15 años de la escuela de Futaleufu en el año 2014. Modulo Dental JUNAEB. Chile. 2014. Documento disponible

10 Oropeza A, Molina N, Castañeda E y cols. Caries dental en primeros molares permanentes de escolares de la delegación Tláhuac. Revista ADM. 2012. LXIX (2); Documento disponible

11 Villavicencio E. El tamaño muestral en tesis de post grado. ¿Cuantas personas debo encuestar? researchgate [Internet]. 2016 [citado 31 Ags 2016]; pag 1-4. Documento disponible 12 Villavicencio E, et al. Pasos para la planificación de una investigación clínica. oactiva.ucacue.edu.ec. 2016; 1(1):1-5. Documento disponible

13 Petersen P. Oral Health Surveys Basic Methods. 5th Edition. France: World Health Organization 2013. Implementing the survey. P29-34

14 Villavicencio E. Proyecto: Prevalencia de Caries en escolares de 12 años en Cuenca 2016. researchgate.net Documento disponible 